\title{
Reversible anabolic androgenic steroid-induced cardiomyopathy
}

\author{
Jessica Joseph ${ }^{1 *}$, Syed Yaseen Naqvi ${ }^{1}$, and Eron Sturm ${ }^{2}$ \\ ${ }^{1}$ Department of Internal Medicine-Pennsylvania Hospital, University of Pennsylvania, USA \\ ${ }^{2}$ Department of Interventional Cardiology-Pennsylvania Hospital, University of Pennsylvania, USA
}

\begin{abstract}
A 33-year-old caucasian male presented to the emergency department with lower extremity edema, dyspnea, and orthopnea. A transthoracic echocardiogram demonstrated an ejection fraction of $10 \%$ with global hypokinesis, biventricular dilatation, and moderate mitral regurgitation. A right heart catheterization demonstrated a pulmonary capillary wedge pressure of $40 \mathrm{mmHg}$ and reduced cardiac index of $1.7\left(\mathrm{~L} / \mathrm{min} / \mathrm{m}^{2}\right)$. Coronary angiography revealed no evidence of obstructive coronary artery disease. An extensive infectious and rheumatologic workup was negative. During the clinical admission for New York Heart Association(NYHA) class 4 heart failure, the patient developed flash pulmonary edema causing hypoxic respiratory failure and he was subsequently intubated requiring ionotropic support. He received aggressive IV diuresis and was eventually weaned off ionotropic support. Once extubated the patient admitted to using anabolic androgenic steroids for the past year. At 10-month cardiology follow-up, continuation of standard medical therapy for systolic heart failure, and complete cessation of anabolic androgenic steroids usage, the patient remained asymptomatic with significant improvement of his ejection fraction to $45 \%$ making anabolic androgenic steroid-induced cardiomyopathy the most likely diagnosis.
\end{abstract}

\section{Introduction}

Acute heart failure is defined as the onset of symptoms or signs of heart failure (HF) in a patient with no prior history of heart failure. It is uncommon to have acute decompensated heart failure in patients without concomitant acute coronary syndromes [1]. Acute decompensated heart failure broadly represents new or worsening symptoms or signs of dyspnea, fatigue or edema that leads to hospital admission or unscheduled medical care that are consistent with an underlying worsening of left ventricular function [2]. It is estimated that 5.7 million Americans greater than 20 years of age have HF [3]. Currently there are 870,000 new HF cases diagnosed annually [3]. It is projected that the prevalence of HF will increase $46 \%$ from 2012 to 2030 , resulting in greater than 8 million people over 18 years of age with HF [4]. Symptoms of HF are the result of severe pulmonary congestion due to elevated left ventricular filling pressures which can occur with or without low cardiac output. Acute HF can occur in patients with preserved or reduced ejection fraction [3]. Usual causes are conditions such as coronary heart disease, hypertension, valvular heart disease, atrial arrhythmias, and/or noncardiac conditions (including renal dysfunction, diabetes, and anemia) [3].

\section{Case report}

A 33-year-old caucasian male with a past medical history of asthma presented from an outside hospital with progressive lower extremity edema, dyspnea initially on exertion then at rest, and orthopnea. Patient stated that his symptoms began 2 weeks prior to admission. Previous to this, the patient was an active weight-lifter and attended the gym 6 days per week. At presentation, his vital signs were blood pressure of 145/90, heart rate of 133 beats per minute, respiratory rate of $35-40$, oxygen saturation of $95 \%$ on 2 liters nasal cannula, and he was afebrile. On physical exam, the patient was in respiratory distress with accessory muscle use, and paradoxical breathing. Lung exam was remarkable for diffuse rales and his legs had 2 plus pitting edemas bilaterally up to both his knees.

Laboratory investigations demonstrated elevated erythrocyte sedimentation rate and d-dimer. His infectious work-up included cytomegalyvirus, ebstein-barr virus, human immunodeficiency virus, respiratory viral panel, hepatitis virus $\mathrm{B} / \mathrm{C}$, coxsackie $\mathrm{A}$ virus, parvovirus $\mathrm{B} 19$, and blood cultures which were all negative. Urine drug screen was negative. His rheumatological work-up included anti-nuclear antibody, anti-smooth muscle antibody, and anti-mitochondrial antibody were within normal limits. His troponins were trended and were all negative. Other investigations including blood count, thyroidstimulating hormone, liver function, cholesterol level, ferritin were all within normal limits.

Chest $\mathrm{x}$-ray demonstrated that his heart was enlarged with diffuse patchy opacities and pulmonary vascular cephalization consistent with moderate pulmonary edema. Electrocardiogram demonstrated sinus tachycardia with a heart rate of 125 beats per minute (Figure 1). A transthoracic echocardiogram (TTE) from an outside hospital demonstrated acute systolic heart failure with left ventricular ejection fraction of $10 \%$ with global hypo kinesis with no evidence of hypertrophic obstructive cardiomyopathy. A repeat TTE on hospital

Correspondence to: Jessica Joseph, Department of General Medicine Division, Pennsylvania Hospital, 800 Spruce Street, 1 Pine West, Philadelphia PA, 19106, Email: Jessica.joseph@uphs.upenn.edu

Key words: anabolic androgenic steroids, acute heart failure

Received: January 19, 2017; Accepted: February 16, 2017; Published: February 18,2017 


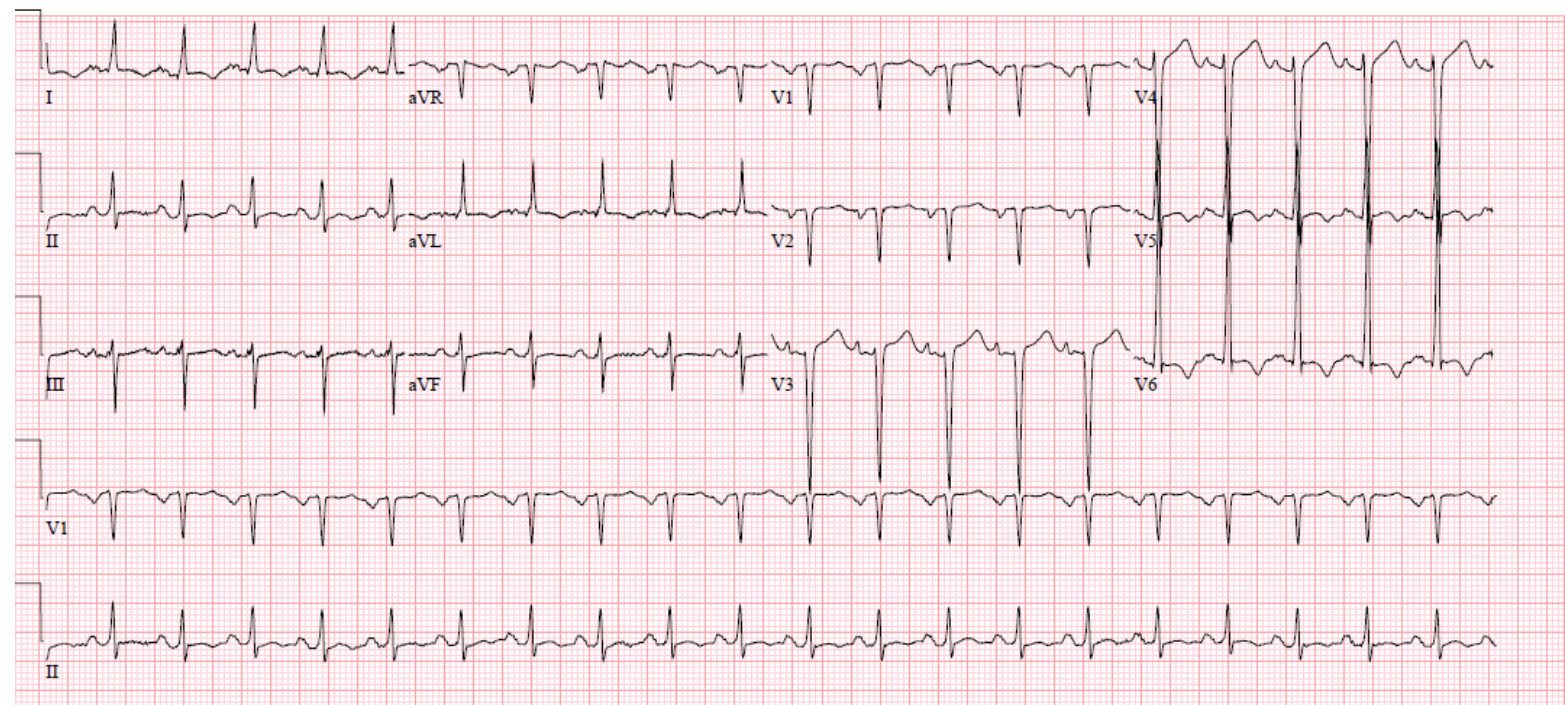

Figure 1. EKG demonstrating sinus tachycardia with a rate of 125 . Normal axis, PR, QRS, and QT intervals. Left ventricular hypertrophy, septal wall infarct of age undetermined age, and inverted T wave abnormality in V5-V6 which could signify lateral ischemia.

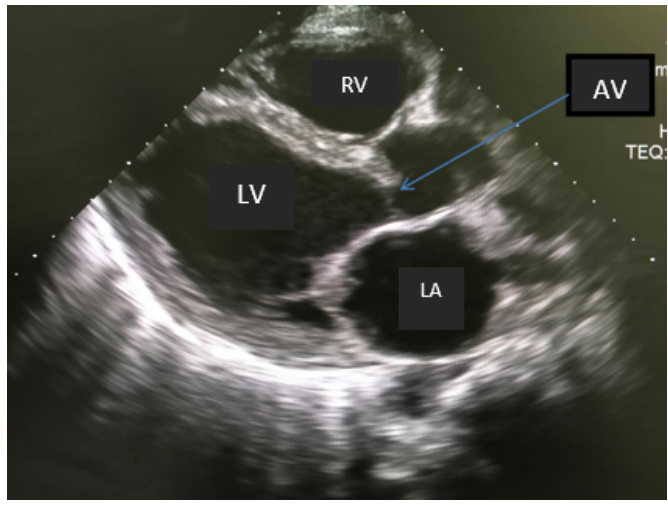

Figure 2. Parasternal long axis view from TTE. This echo images showed a mildly dilated left atrium (LA) and a mildly left ventricle (LV). Aortic valve (AV), right ventricle (RV).

day 4 demonstrated an ejection fraction of less than $25 \%$, severe global hypokinesis of the left ventricle consistent with a cardiomyopathy, moderate biatrial/biventricular dilation and moderate mitral valve regurgitation (Figures 2 and 3). Cardiac catheterization was performed on hospital day number 6 . The right heart catheterization showed elevated pulmonary capillary wedge pressure of $40 \mathrm{mmHg}$ and reduced cardiac index of $1.7\left(\mathrm{~L} / \mathrm{min} / \mathrm{m}^{2}\right)$ where as the left heart catheterization was negative for coronary artery disease. A cardiac magnetic resonance imaging was not suggestive of infiltrative diseases.

Given his worsening clinical status, the patient was scheduled to be transferred to a tertiary center for urgent evaluation for placement of a mechanical left ventricular assistance device, but he decompensated the evening prior. His chest $\mathrm{x}$-ray demonstrated worsening pulmonary edema. He then became lethargic, tachypneic, and was intubated due to flash pulmonary edema causing hypoxic respiratory failure. He was stabilized on norepinephrine and milrinone drips and was transferred to a tertiary facility. Ultimately, his norepinephrine and milrinone drips were weaned off and he was transitioned to an oral heart failure regimen including metoprolol and enalapril. The etiology of his cardiomyopathy was unclear but the patient endorsed that he also had been using anabolic androgenic steroids (AAS) for one year, which was supplied by a friend from his gym. Thus, in light of his otherwise

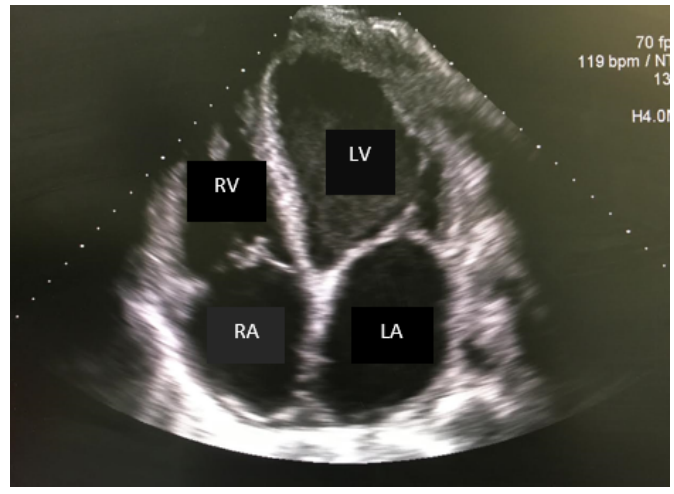

Figure 3. Four chamber view of TTE. Echo image demonstrates biatrial and biventricular dilation. Right atrium (RA), left atrium (LA), left ventricle (LV), right ventricle (RV).

extensive negative workup, it was postulated that the abuse of AAS was the likely cause of his cardiomyopathy. At the time of hospital discharge, he had NYHA class 2 CHF symptoms and he was able to ambulate without any difficulty. He was instructed not to resume taking AAS.

At 10-month cardiology follow-up with continuation of standard medical therapy for systolic heart failure and complete cessation of AAS usage, the patient remained asymptomatic with significant improvement of his ejection fraction to $45 \%$. He currently has NYHA class 1 symptoms.

\section{Discussion}

The use of AAS has been linked to causing myocardial infarction, sudden cardiac death, ventricular fibrillation with exercise, atrial fibrillation, cardiac tamponade, and development of dilated cardiomyopathy [5]. AAS like other endogenous steroids influence left ventricular hypertrophic response through the androgen receptor. Androgen receptors are found on skeletal muscle and also on cardiac myocytes. They cause alterations in heart structure, including left ventricular hypertrophy, dilation which can cause impaired contraction and relaxation [6]. Anabolic steroids can induce an unfavorable enlargement and thickening of the left ventricle, which loses its diastolic 
properties with the mass increase [7]. AAS can also cause hypertension, dyslipidemia, and impaired fasting glucose [8]. The effect of AAS on the cardiovascular system has been well described in the literature; however, the knowledge of these effects amongst amateur sports participants is poor [9]. A qualitative, cross-sectional, observational study using an anonymous online questionnaire completed by amateur sports participants enrolled in competitive sports teams at an Irish university demonstrated that one-third of respondents personally knew someone that uses anabolic steroids, however $59 \%$ of participants were never advised about their health effects [9]. The majority (59\%) of respondents obtained their nutritional advice from internet sites or friends, only $26 \%$ of respondents knew about the blood pressure, and cholesterol effects of anabolic steroid use [9].

The knowledge of the cardiac risks associated with the use of AAS amongst sports participants is generally poor [9]. Efforts must be made to educate coaches and gym facilities about the harmful effects of certain performance enhancing drugs.

\section{Conflict of interest}

None

\section{References}

1. Allen LA, O'Connor CM (2007) Management of acute decompensated heart failure. CMAJ 176: 797-805. [Crossref]

2. Felker GM, Adams KF Jr, Konstam MA, O'Connor CM, Gheorghiade M (2003) The problem of decompensated heart failure: nomenclature, classification, and risk stratification. Am Heart J 145: S18-25. [Crossref]

3. Mozaffarian D, Benjamin EJ, Go AS, Arnett DK, Blaha MJ, et al. (2015) Heart disease and stroke statistic--2015 update: a report from the American Heart Association. Circulation 2015;131: e29-e322. [Crossref]

4. Heidenreich PA, Albert NM, Allen LA, Bluemke DA, Butler J, et al. (2013) Forecasting the impact of heart failure in the United States: a policy statement from the American Heart Association. Circ Heart Fail 6:606-619. [Crossref]

5. Ahlgrim C, Guglin M (2009) Anabolics and cardiomyopathy in a bodybuilder: case report and literature review. J Card Fail 15: 496-500. [Crossref]

6. Figueredo VM (2011) Chemical cardiomyopathies: the negative effects of medications and nonprescribed drugs on the heart. Am J Med 124: 480-488. [Crossref]

7. De Piccoli B, Giada F, Benettin A, Sartori F, Piccolo E (1991) Anabolic steroid use in body builders: an echocardiographic study of left ventricle morphology and function. Int J Sports Med 12: 408-412. [Crossref]

8. American College of Sports Medicine position stand on the use of anabolic-androgenic steroids in sports. (1987) Med Sci Sports Exerc 19: 534-539. [Crossref]

9. Naqvi SY, Flaherty G (2016) Knowledge, and Attitudes of Amateur Sports Participants Regarding the Cardiac Risks Associated with the Use of Anabolic-Androgenic Steroids. IOSR Journal of Sports and Physical Education 36-44.

Copyright: (C2017 Joseph J. This is an open-access article distributed under the terms of the Creative Commons Attribution License, which permits unrestricted use, distribution, and reproduction in any medium, provided the original author and source are credited. 